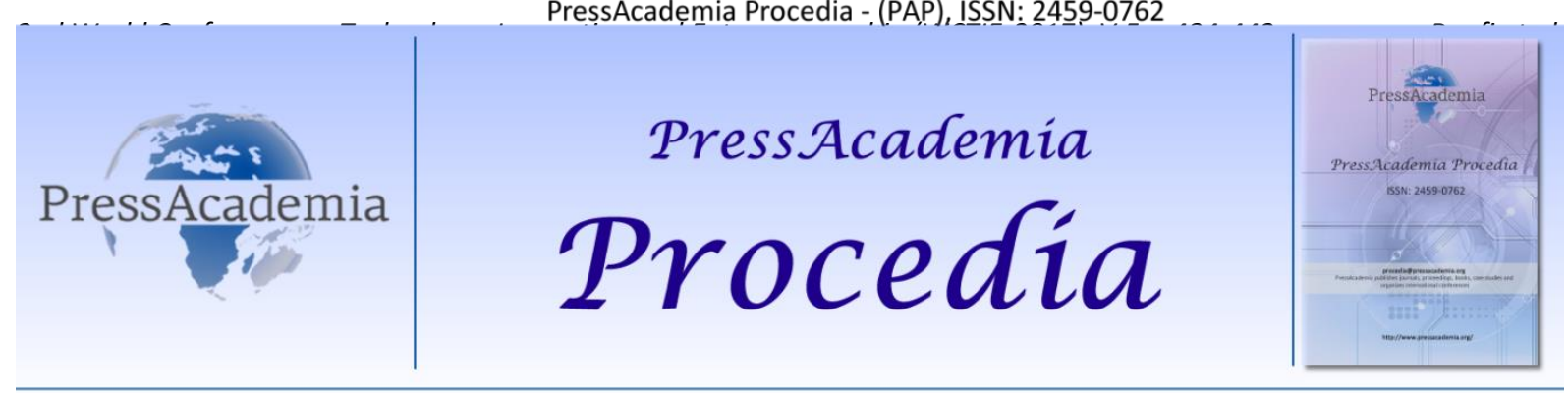

2nd World Conference on Technology, Innovation and Entrepreneurship

May 12-14, 2017, Istanbul, Turkey. Edited by Sefer Şener

\title{
MODELING A CONTEXT-AWARE FRIEND-OF-A-FRIEND(FOAF) APPLICATIONS FOR MOBILE PLATFORMS
}

DOI: 10.17261/Pressacademia.2017.621

PAP-WCTIE-V.5-2017(59)-p.-434-442

\author{
Bujar Raufi ${ }^{1}$, Florije Ismaili ${ }^{1}$, Jaumin Ajdari ${ }^{1}$, Xhemal Zenuni ${ }^{1}$ \\ ${ }^{1}$ South East European University, b.raufi, f.ismaili, j.ajdari, xh.zenuni@seeu.edu.mk
}

\begin{abstract}
We live in an era where the evolution of social networks and developments in the field of information technology witnesses and exponential growth. On a daily basis, we have an ever growing, an ever expanding social network users and as a result we affirm an increase of data that is distributed across different platforms. In this paper, we aim to treat the semantic web from the prism of creating a suitable infrastructure for data integration on the web. One of the most promising applications of the semantic web is the presentation of profiles using an RDF (Resource Description Framework) schema called Friend-of-Friend (FOAF) which represents a machine-processable ontology for describing persons, their activities and relations to other people and objects. In order to demonstrate the concept on how to integrate, relate and share information using FOAF, we model and develop a mobile application called "Find professional" which consists in creating, finding and interacting in a context aware fashion with other users via FOAF. The "Find Professional" mobile app can create a FOAF profile either by asking user's direct input or it can utilize other social network profiles such as Facebook for importing user public profiles into FOAF. User profiles represent RDF files that can be read and queried by semantic query languages like SPARQL, which returns data to their user profiles as well as finds professionals in the FOAF app based on user's geographic location. The application is also evaluated with a field usability testing performed against ten randomly picked user from various profiles and backgrounds. Usability testing process did not yield severe problems during application evaluation and comments are more related to user interface rather than difficulty in user task completion.
\end{abstract}

Keywords: Semantic web, foaf dictionary, semantic social network, context-awareness.

\section{INTRODUCTION}

FOAF (Friend-Of-A-Friend) consists on the connection between people and objects in one side and semantic information on the other using a web platform. FOAF ontology is machine redable and processable which serves to describe activities and their relationship with other persons and objects as well as can be used for entity self-description. The role of FOAf is threefold, first it integrates social networks for human collaboration, friendship and association; second it integrates the so called representational networks which gives a simplified view of a universe based on factual terms, and third it integrates information networks that utilize the various types of linkings in the Semantic Web to share independently published descriptions of resources on this inter-connected world (Brickley, 2016). Management from the perspective of the social network integration that are distributed in different profiles is seen as a very profitable union of all these relationships in a single set of data. In an integrated social networks, persons who have several user profiles will be presented as a single entity. Information about users which are distributed accross various user profiles will also be interconnected and joined. This paper presents the importance of FOAF vocabulary components as well as conduct an analysis on how these components enable data integration in a single mobile platform.

As a result, all theoretical analysis based on earlier scientific research and W3C specifications about semantic web and FOAF technologies as pillars of supporting this platform will be conceptualized and developed through an android application. The application consists in creation of social network linkings as well as their localization and matching of similar profiles. This application uses simple forms where users can create their FOAF profile as well as integrate an approach that aims to 
join Facebook application data that can be added to a FOAF profile as RDF. The application generates the RDF file that can be read and queried by specialized languages such as SPARQL (SPARQL, 2016), which returns data to their user profiles. The paper focuses also on examination of all necessary classes and FOAF vocabulary items (such as components and the persons he or she knows), expressed in RDF format. All this is achieved through a mobile application that converges all the aspects of FOAF usage in one context-aware environment.

The rest of this paper is organized as follows: section 1 introduces related work done with relation to FOAF based mobile applications, section 3 introduces the FOAF mobile application from the sense of its architecture and functionality, section 4 evaluates the application with findings from usability testing and section 5 concludes the paper with some outlines for future improvements.

\section{RELATED WORK}

The proliferation of the web made it possible for users to visit different sites, read the information published there and interact with the web in various ways. In recent years, new types of applications emerged which allow visitors not only to read the information passively, but also to act and influence upon user's activities, such as the online reservations, ticketing services and recommender systems among others.

Web applications are being exponentially populated and as a result information is stored in huge databases that utilize various technologies for that purpose such as Datacubes (Edoh-Alove, 2016), (Höffner, 2016) or noSQL databases (Swaminathan, 2016), (Gantz, 2016). But the information stored in these databases are either semi-relational, un-relational (in case of noSQL) or hidden from user activities and existing search engines. Even though that search engines are used to find and collect information, most of the time they fail in finding a direct and accurate information to whom users are interested (Dan \& Davidson, 2016). The information retrieval process that lies out of the scope of semantic web is facing serious challenges that influence the search process in many ways. Problems that IR is facing are ranging from spams, poor content quality, lack of quality evaluation, absence of web conventions up to presence of duplicate sources and vagely structured data and they all represent obtrusive factors for a good search (Henzinger, 2002). Based on the above mentioned, the approach for the future would be to structure the data as much as possible, together with their association with the additional information that may be descriptive or helpful to end users of the web (Heath, 2011). This is the core issue which semantic web faces. Semantic search helps in finding information by dealing with complex queries, presenting precise and self-explanatory results and quick response (Lei, 2006). It also provides a common carrier structure, which allows the distribution and reuse of data between applications, enterprises and community boundaries beyond (BernersLee, 2016), (Torres, 2017). The goal is to allow communication between the automated processes, which make the data readable by machines in a detailed way and helps in finding information on millions of subjects in their web pages. In order to achieve the goal, it is encouraged to develop web applications, especially in the mobile application area that will allow users to enter semantically structured data that can be later reused by semantic agents.

In many approaches, challenges in creating an appropriate infrastructure for the process of integration of information on the web is risen but not holistically addressed (Allemang, 2011). In Meng et. al, an analysis of the problem of knowledge base semantic integration using crowd intelligence is elaborated. As a result, a novel hybrid framework for knowledgebase semantic integration considering the semantic heterogeneity of such KBs class structures is introduced. The proposal also addresses the problem of vastnes of such knowledgebases as well as problems that cowdsourcing introduces. The approach, faces the challenges of crowdsourcing as well as does not provide a unified format of data representation of such integration. Another approach proposes the use of Bluetooth technologies to connect multiple mobile devices in an ad hoc network in a user-centered location-based service that enables stable and efficient data communication (Jiang, 2007). However the lack of the approach is in the underlying infastructure of the Bluetooth which does not support large scale infrasturture that would allow social network data integration.

The objective of this paper is to build a semantic social network based on FOAF ontology. There are various approaches that deal with integration of semantic web into different web and mobile platforms. Many of the attempts are made in the field of Linked Open Data. Some of the ideas involve creating a user friendly interfaces with relation to navigation of the resources in Linked Open Data Repositories (Bizer et. al, 2009), (Becker, 2008). In Becker et. al, a mobile application is proposed which presents an overview of the Geospatial Semantic Web and introduces DBpedia Mobile, a location-aware Semantic Web client which is an app that can be used in many mobile platforms (Becker, 2009). Context awareness is an important aspect when it comes to mobile application and search of semantic repositories. It enables computer systems to anticipate users' needs and to act in advance in one hand and improve the user interactions with computing system on the other (Chen, 2000).

To the best of our knowledge, there are many applications and architectures that tend to bring social networking paradigm to mobile platform in one holistic design (Rana, 2009). We can mention few of them as: (Sheth, 2009), (Schaffert, 2009), 
(Jung, 2010), (Torres, 2017) as well as some industry approaches such as (Serena, 2017) . However they lack proper data integration from social networks as well as their applicability in the domain of context-awareness in such cases.

In this paper we integrate all of the above mentioned by developing a mobile social network application called "Find professional" which consists in utlizing other social network profiles for importing and finding professionals in FOAF based on their geographic location.

\section{FRIEND-OF-A-FRIEND (FOAF) APPLICATION FOR MOBILE PLATFORMS}

In order to demonstrate the functionality of FOAF and its application to social networking, we have introduced a small app that builds upon the above mentioned ideas and it tends the integration of profiles from other social network accounts in order to create a FOAF user profile for finding various professionals.

The FOAF ontology is the most widely used domain ontology on the semantic web. Many people are using it in an open and extensible fashion by defining new classes and properties to use with FOAF (Ding et. al, 2004). The FOAF vocabulary in itself includes classes and properties found useful to describe people online. It is interoperable with other semantic web standards as well as support auto discovery and cross reference with other FOAF documents. It can be serialized in various support formats for semantic web such as: RDF / XML, RDFa, Microdata, N3, N-Triples or JSON. The following example depicts a FOAF user represented as JSON serialization.

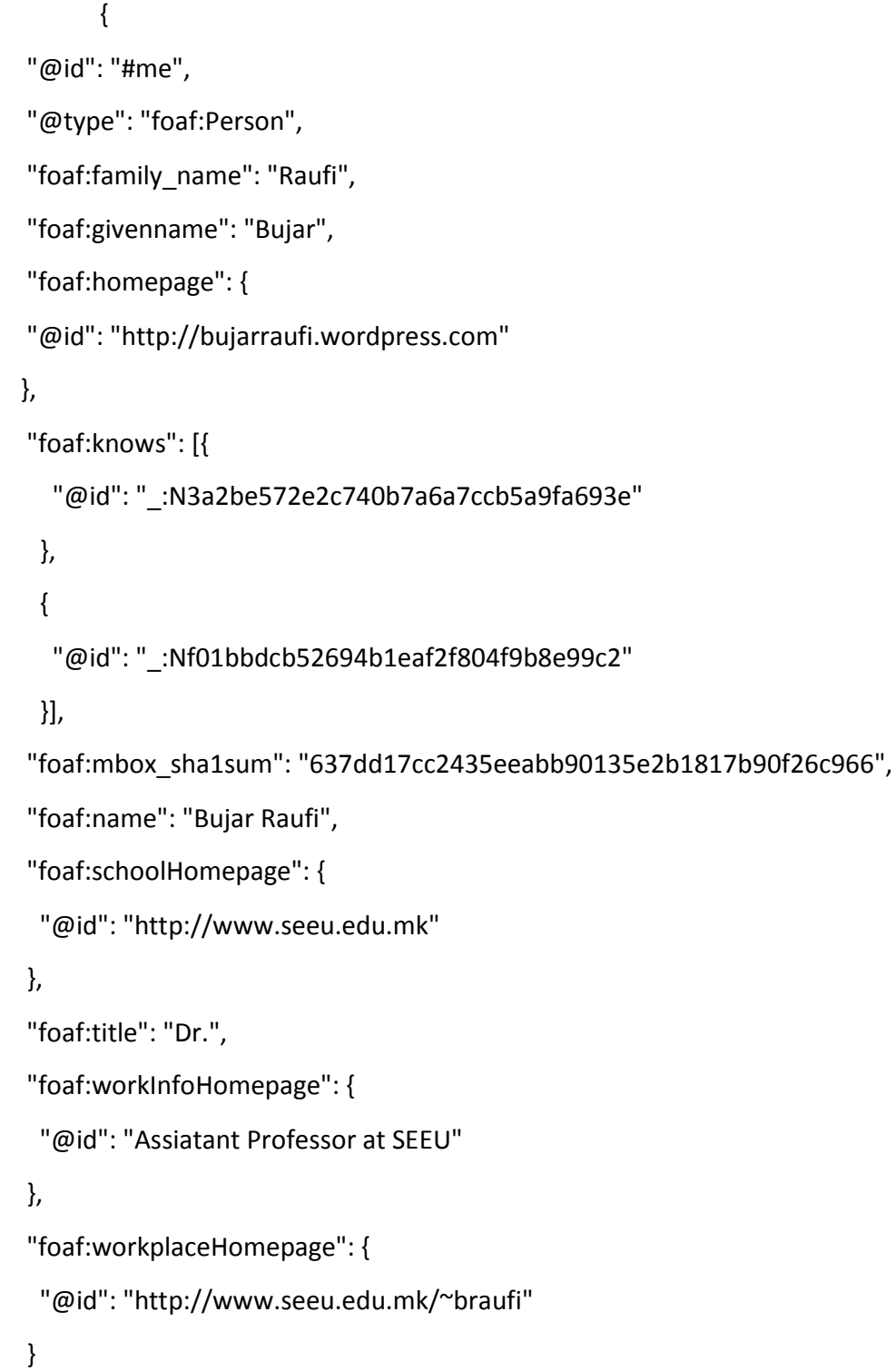




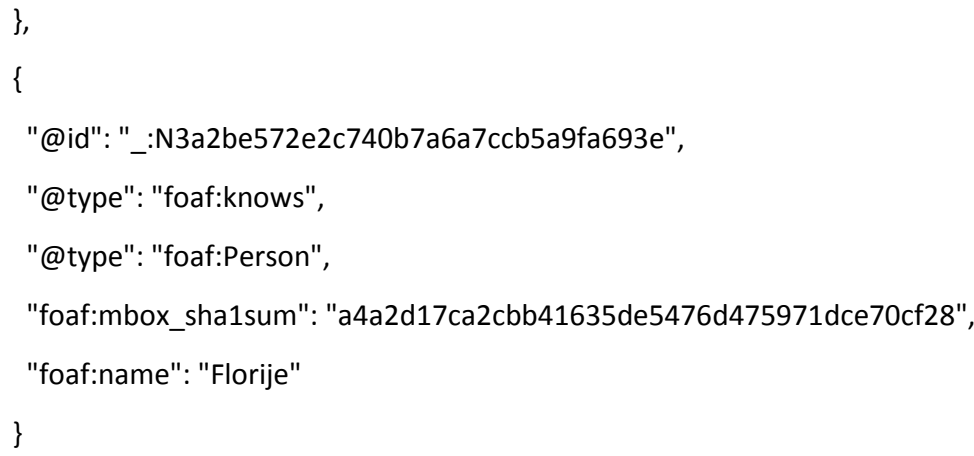

\section{Figure 1: JSON Serialization of a FOAF Profile}

Context-aware systems and context aware computing represent a general class of mobile systems that can sense their physical environment, and adapt their behavior accordingly (Robles, 2010), (Thyagaraju, 2012). Context-aware is used in many applications for purpose to include nearby people, devices, lighting, noise level, network availability, and even in social situations, such as whether a person is with its family or friends or in finding people with similar preferences in vicinity (Robles, 2010). For the design of the application the context-awareness approach is used for allowing access to find similar profiles based on user geographical location as well as their profession.

\subsection{Application Architecture}

The main components of the application are treated in conjunction with user's interaction with the system. The overall logic of the application is given through the following main actions:

- If a user does not possess an actual FOAF profile. The "Find Professional" app will allow users to create new accounts, data can be also retrieved from their other social network accounts (such as Facebook). The data necessary for creating a Find professional FOAF profiles are:

- Name

- Last name

- Facebook ID

- Birthday

- E-mail

- The information about user's birthday and e-mail address can be taken user's accounts if they are publicly available. If a user does not have a Facebook account, a regular registration of a profile goes in the application. Fig 2. Illustrates the initial four interfaces of the Find Professional mobile app.

Figure 2: Initial Interface of the Mobile App
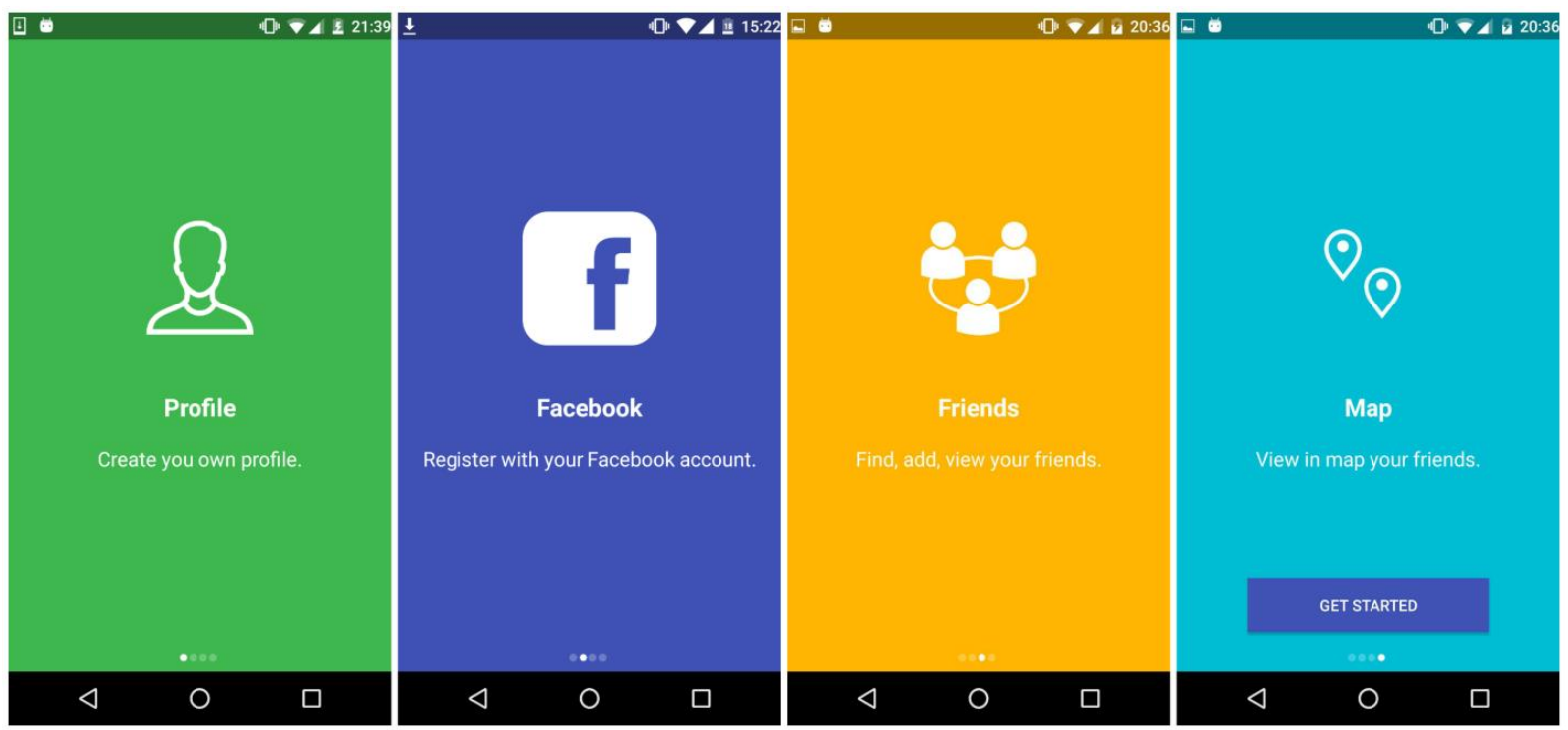
- $\quad$ By sliding the page on the left we will receive different pages' base on the stages of our registration process. I.e. either to login via Facebook or create a new account. If we already have e Facebook account, after login the initial data will be populated to a FOAF profile for creating our Find Professional profile as well as search for created profiles. These interfaces are illustrated in Fig. 3.

Figure 3: User Interface during Login and Search

\begin{tabular}{|c|c|c|c|c|}
\hline$\square 0 \quad 0 \times 4020: 36$ & $\square \pm \quad 0 \times 4 \div 15: 22$ & प्व & $\nabla 4=12: 40$ & OP/ 20:38 \\
\hline FOAF & $\leftarrow$ & $\leftarrow$ Enter your FOAF & & $\equiv$ Home \\
\hline Username & Enter vour FOAF & Username & & $\because$ :- Genc Arifi \\
\hline Password & Name & & & $\ddot{\because 0}$ : Kaltrina Bega \\
\hline$\square$ Remember me? & Lastname & & & :- Donjeta Luma \\
\hline 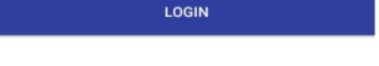 & Emall & & & :-: Egzona R. \\
\hline & $\begin{array}{l}\text { Birthday } \\
\text { Open calendar }\end{array}$ & & & $\because$ : $\quad$ test egzona \\
\hline Register - or - $f$ Log in with Facebook & $\begin{array}{c}\text { Gender } \\
\text { Male } \\
\text { Website }\end{array}$ & & & Ö:- Bujar Raufi \\
\hline & Phone & & FINISH & $\because$ Bujar Raufi \\
\hline 0 & 0 & 0 & ㅁ & 0 \\
\hline
\end{tabular}

The overall user activities are depicted in the use case diagram which is illustrated in Fig 4

Figure 4: Use Case Diagram for "Find Professional" Mobile App

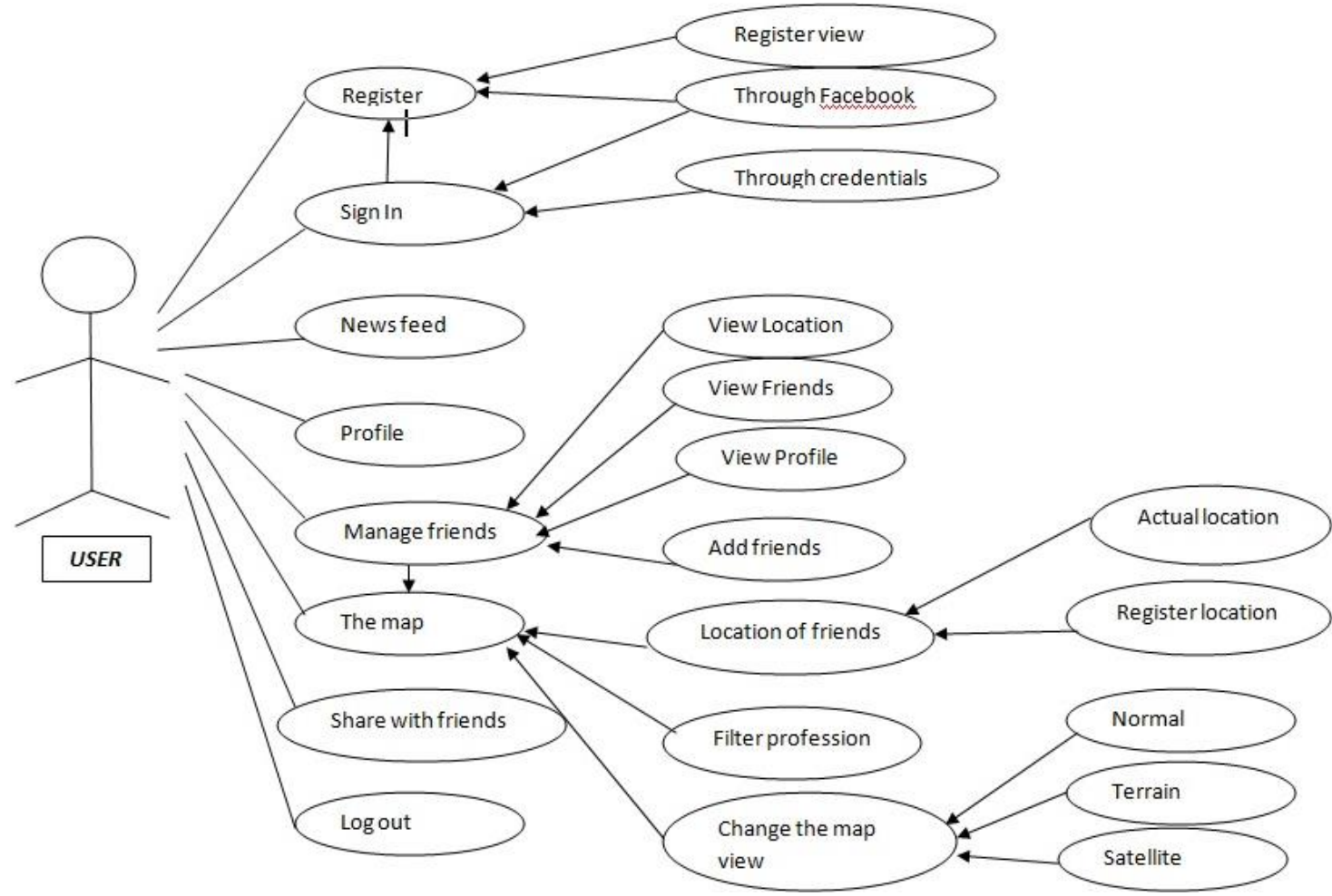




\subsection{Context-Awareness in FOAF Application}

The aspect of context-awareness has been achieved through the geographical location of users that share the same or similar profession and is registered via the application. The model is based on ontological context awareness because the application uses the foaf:based_near property. The application lists nearby friends, their names and professions. Fig. 5 illustrates the physical context-awareness as seen from the application interface.

Figure 5: Maps and Appearance of Friend's Locations

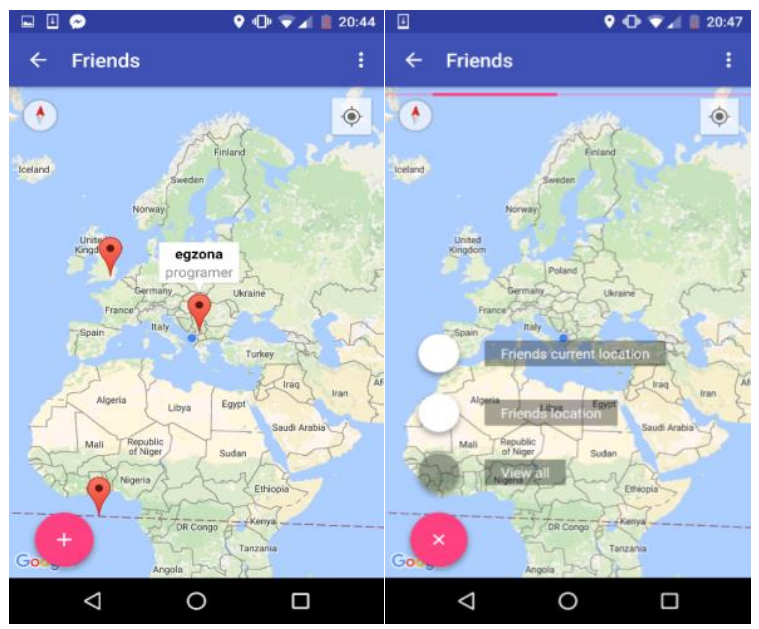

\section{MOBILE PLATFORM EVALUATION}

The evaluation process of the application was performed through a usability testing of the mobile platform. Usability testing represents a common tool which is used to evaluate the usability of a mobile application in a development phase. Usability testing are controlled in environments using a think aloud protocols initially set in a pioneering work of K. A. Ericsson and H. A. Simon's $(1980,1984)$ (Kaikonnen et al., 2005). For the evaluation purposes, we favoured the field tests against stricter laboratory test. We considered that field tests are more suitable when evaluating usability of mobile applications, considering that the context affects usage and performance. This was also hypothesised and favoured in (Kaikonnen et al., 2005). The procedure of evaluation of the mobile platform followed a strict procedure as described in (Sun et. al, 2013).

\subsection{Setup}

The study took place on the street by presenting the application to ordinary users and briefing them shortly about what an application was about. This user experience actions comprised tasks that were given after the application briefing occured and most of tested users were unfamiliar with each other. The user experience was therefore typical of that encountered during causal and unformal interviews. Information was collected on the site where a short survey was fullfilled.

\subsection{Experimental Scenarios}

The successful uilization of scenarios takes into account the diversity of contexts encountered by users. The scenarios where used in the application presented in the form of tasks that should be fullfilled by the user. Tasks that were given to users in different contexts are as follows:

1) Getting started: Download and Install FOAF Application

2) Finding and opening the FOAF application

3) Open a FOAF Profile through Facebook, if no account available Register via application

4) Navigating the main menu functionalities

5) Searching for other FOAF profiles

6) Looking up for nearby profiles based on the same profession

7) Utilizing the map for finding the similar users based on professions

8) Closing the application

Besides these tasks, we have also introduced some intermediary subtasks that varied from user to user. 


\subsection{Participants}

Ten participants were recruited at a random basis. Their ages ranged from 24 to 55 years, with a mean of 29.7, and as much as equal gender split. A range of occupations were represented, including accountant, mechanics, computer scientist, architect, SEO Expert, and Higher Education professional; four participants were university students. Recruitment criterion was the possesion of a smartphone longer than a year.

\subsection{Field Study Results}

The field experiments generated substantial amounts of rich and valuable data in relatively short time. It is worth mentioning that participants in the testing emphasized the problems of mobile usage rather than simply application usability, and typically those problems were expressed in the form of occasional language. To the participants, the field experiment environment felt moderately informal, and the users talked freely about the use of the application and their sentiments. Users expressed how the field experiment granted them the relaxed look and feel and the possibility to communicate with the researcher as they undertook the evaluation scenarios. For example, users were concerned about the interface such as colors, background and colour vividness. Even though, there were cases where users generally expressed broader views and were able to give a wide range of evaluation-related information during the experiment, such as expressing ideas related to requirements concerning context awareness. A particular example this sort of data generated during the field study was the need for the mobile content and delivery to be highly integrated into the temporal flow of the searching proceess while finding similar matches based on profession. After the experiment, users filled a survey where they had to evaluate each task from 1 to 5, where 1-indicating poor and 5-indicating excellent experience. The detailed list of usability testing for each task i given as in Table 1.

Table 1: Usability Testing on Field Users, Their Task and Requirements

\begin{tabular}{llllllllll}
\hline Name & Age & Task 1 & Task 2 & Task 3 & Task 4 & Task 5 & Task 6 & Task 7 & Task 8 \\
\hline User1 & 55 & 5.00 & 5.00 & 5.00 & 5.00 & 4.00 & 3.00 & 2.00 & 5.00 \\
User2 & 25 & 5.00 & 5.00 & 5.00 & 5.00 & 5.00 & 5.00 & 5.00 & 5.00 \\
User3 & 24 & 5.00 & 5.00 & 5.00 & 5.00 & 5.00 & 5.00 & 5.00 & 5.00 \\
User4 & 25 & 5.00 & 3.00 & 2.00 & 5.00 & 5.00 & 4.00 & 3.00 & 4.00 \\
User5 & 30 & 5.00 & 5.00 & 5.00 & 4.00 & 5.00 & 5.00 & 5.00 & 5.00 \\
User6 & 26 & 5.00 & 2.00 & 3.00 & 4.00 & 5.00 & 4.00 & 5.00 & 5.00 \\
User7 & 25 & 5.00 & 5.00 & 5.00 & 5.00 & 5.00 & 5.00 & 5.00 & 5.00 \\
User8 & 24 & 5.00 & 5.00 & 5.00 & 5.00 & 5.00 & 5.00 & 5.00 & 5.00 \\
User9 & 25 & 5.00 & 5.00 & 5.00 & 5.00 & 5.00 & 5.00 & 5.00 & 5.00 \\
User10 & 38 & 4.00 & 3.00 & 5.00 & 5.00 & 5.00 & 5.00 & 5.00 & 4.00 \\
\hline
\end{tabular}

The user experience assesment was conducted against a paper based detailed design of the mobile prototype application as control measurement. To ensure that the user interface itself (rather than prior knowledge of the user) was not majorly influencing the experiment results, the user-initiated interface tasks were evaluated by calculating the percentage of tasks completed by participants and analysing user comments and matching them against detailed design protoytype taken as a measurement control. Table 2 depicts the overall user experience assesment for each task given in experimental scnearios. The methodology followed is used in (Grawe, 2016).

Table 2: Overall User Experience Evaluation for Each Task

\begin{tabular}{llll}
\hline \multicolumn{1}{c}{ Task } & & $\begin{array}{c}\text { Friedman } \\
\text { (8 related Samples) }\end{array}$ & Multiple paired comparisons $\boldsymbol{\alpha}=\mathbf{0 5}$ \\
\hline $\begin{array}{l}\text { Getting started: Download and Install FOAF } \\
\text { Application }\end{array}$ & $\begin{array}{l}\chi^{2}(7)=49.0, \\
P<.002\end{array}$ & $N=10,\left|R_{\text {paper }}-R_{\text {prototype }}\right|=1.0^{*}$ \\
Finding and opening the FOAF application & $\begin{array}{l}\chi^{2}(7)=43.0, \\
P<.002\end{array}$ & $N=10,\left|R_{\text {paper }}-R_{\text {prototype }}\right|=7.0^{*}$ \\
Open a FOAF Profile through Facebook, if no & $\begin{array}{l}\chi^{2}(7)=45.0, \\
P<.002\end{array}$ & $N=10,\left|R_{\text {paper }}-R_{\text {prototype }}\right|=5.0^{*}$ \\
account available Register via application & &
\end{tabular}


Navigating the main menu functionalities

Searching for other FOAF profiles

Looking up for nearby profiles based on the same profession

Utilizing the map for finding the similar users based on professions

Closing the application

$$
\begin{array}{ll}
\chi^{2}(7)=48.0, & N=10,\left|R_{\text {paper }}-R_{\text {prototype }}\right|=2.0^{*} \\
P<.002 & \\
\chi^{2}(7)=49.0, & N=10,\left|R_{\text {paper }}-R_{\text {prototype }}\right|=1.0^{*} \\
P<.002 & \\
\chi^{2}(7)=46.0, & N=10,\left|R_{\text {paper }}-R_{\text {prototype }}\right|=4.0^{*} \\
P<.002 & \\
\chi^{2}(7)=45.0, & N=10,\left|R_{\text {paper }}-R_{\text {prototype }}\right|=5.0^{*} \\
P<.002 & \\
\chi^{2}(7)=48.0, & N=10,\left|R_{\text {paper }}-R_{\text {prototype }}\right|=2.0^{*} \\
P<.002 &
\end{array}
$$

* Indicates a insignificant difference between user experience tasks done in the experiment and those expected in the control values user activities in the paper detailed design

From the overhead data it can be seen clearely that the usability testing process did not yield any severe problems during application evaluation and comments are more related to user interface rather than difficulty in user task completion.

\section{CONCLUSION AND FUTURE WORK}

In this paper we introduced a social network based mobile application that utilized the FOAF ontology for profile creation, navigation and profile matchmaking and finding based on professions given in those profiles. The application was evaluated with a usability testing performed against ten field users. The results indicated no severe problems in using the mobile application.

Future research directions would involve:

- Creating FOAF profiles by migrating other social network accounts such as Twitter and Linkedln and incorporating in the application. Linkedln accounts would be the next logical step considering that it represents a social network where professionals share their expertise.

- Using and linking to other semantic data such as DBPedia and widening the search and reasoning over the semantic data retrieved.

We firmly consider that applications of this type in the future would yield semantically enriched data to be widely available on the web and as a result, it will bring the mobile platforms closer to Linked Open Data in particluar and Semantic Web in general.

\section{REFERENCES}

Allemang, D., \& Hendler, J. (2011). Semantic Web for the Working Ontologist: Effective Modelling in RDFS and OWL. Elsevier.

Becker, C., \& Bizer, C. (2008). DBpedia Mobile: A Location-Enabled Linked Data Browser. Ldow, 369, 2008.

Becker, C., \& Bizer, C. (2009). Exploring the geospatial semantic web with dbpedia mobile. Web Semantics: Science, Services and Agents on the World Wide Web, 7(4), 278-286.

Berners-Lee, T., \& Jaffe, J. (2016). W3C DATA ACTIVITY Building the Web of Data. Retrieved February 15, 2017, from https://www.w3.org/2013/data/

Bizer, C., Heath, T., \& Berners-Lee, T. (2009). Linked data-the story so far. Semantic services, interoperability and web applications: emerging concepts, 205-227.

Brickley, D., \& Miller, L. (2016). The Friend Of A Friend (FOAF) vocabulary specification, November 2007. URL http://xmlns. com/foaf/spec

Chen, G., \& Kotz, D. (2000). A survey of context-aware mobile computing research (Vol. 1, No. 2.1, pp. 2-1). Technical Report TR2000-381, Dept. of Computer Science, Dartmouth College.

Dan, O., \& Davison, B. D. (2016). Measuring and Predicting Search Engine Users' Satisfaction. ACM Computing Surveys (CSUR), 49(1), 18.

Ding, L., Finin, T., \& Joshi, A. (2004). Analyzing social networks on the semantic web. IEEE Intelligent Systems (Trends \& Controversies), 8(6), 815-820.

Edoh-Alove, E., Bimonte, S., Pinet, F., \& Bédard, Y. (2016). New design approach to handle spatial vagueness in spatial OLAP datacubes: application to agri-environmental data. In Geospatial Research: Concepts, Methodologies, Tools, and Applications (pp. 1859-1880). IGI Global.

Gantz, J., \& Reinsel, D. (2016). THE DIGITAL UNIVERSE IN 2020-Big Data, Bigger Digital Shadows, and Biggest Growth in the Far East, IDC IView. 
Grawe, P. H. (2016). Nonparametric Statistics for the Behavioral Sciences. Numeracy: Advancing Education in Quantitative Literacy, 9 (1)

Heath, T., \& Bizer, C. (2011). Linked data: Evolving the web into a global data space. Synthesis lectures on the semantic web: theory and technology, 1(1), 1-136.

Henzinger, M. R., Motwani, R., \& Silverstein, C. (2002, September). Challenges in web search engines. In ACM SIGIR Forum (Vol. 36, No. 2, pp. 11-22). ACM.

Höffner, K., Lehmann, J., \& Usbeck, R. (2016, October). CubeQA-Question Answering on RDF Data Cubes. In International Semantic Web Conference (pp. 325-340). Springer International Publishing.

Jiang, C. (2007). A location-based service provides Bluetooth cell phone user rich content related to objects/people nearby (Doctoral dissertation, New York University).

Jung, J. J. (2010). Integrating Social Networks for Context Fusion in Mobile Service Platforms. J. UCS, 16(15), 2099-2110.

Kaikkonen, A., Kekäläinen, A., Cankar, M., Kallio, T., \& Kankainen, A. (2005). Usability testing of mobile applications: A comparison between laboratory and field-testing. Journal of Usability studies, 1(1), 4-16.

Lei, Y., Uren, V., \& Motta, E. (2006, October). Semsearch: A search engine for the semantic web. In International Conference on Knowledge Engineering and Knowledge Management (pp. 238-245). Springer Berlin Heidelberg.

Meng, R., Chen, L., Tong, Y., \& Zhang, C. (2017). Knowledge Base Semantic Integration using Crowdsourcing. IEEE Transactions on Knowledge and Data Engineering.

Protocol, S. P. A. R. Q. L. (2016). RDF Query Language. RDF Data Access Working Group (DAWG) Std.

Rana, J., Kristiansson, J., Hallberg, J., \& Synnes, K. (2009, July). An architecture for mobile social networking applications. In Computational Intelligence, Communication Systems and Networks, 2009. CICSYN'09. First International Conference on (pp. 241-246). IEEE.

Robles, R. J., \& Kim, T. H. (2010). Review: context aware tools for smart home development. International Journal of Smart Home, 4(1).

Schaffert, S., Eder, J., Grünwald, S., Kurz, T., \& Radulescu, M. (2009, May). KiWi-a platform for semantic social software. In European Semantic Web Conference (pp. 888-892). Springer Berlin Heidelberg.

Serena, F. D. (2017). U.S. Patent No. 9,536,268. Washington, DC: U.S. Patent and Trademark Office.

Sheth, A. (2009). Citizen sensing, social Signals, and enriching human experience. IEEE Internet Computing, 13(4).

Sun, X., \& May, A. (2013). A comparison of field-based and lab-based experiments to evaluate user experience of personalised mobile devices. Advances in Human-Computer Interaction, 2013, 2.

Swaminathan, S. N., \& Elmasri, R. (2016, June). Quantitative Analysis of Scalable NoSQL Databases. In Big Data (BigData Congress), 2016 IEEE International Congress on (pp. 323-326). IEEE.

Thyagaraju, G. S., \& Kulkarni, U. P. (2012). Design and implementation of user context aware recommendation engine for mobile using Bayesian network, fuzzy logic and rule base. International Journal of Pervasive Computing and Communications, 8(2), $133-157$.

Torres, B. P., \& González, A. G. (2017). Evolution of the Semantic Web Towards the Intelligent Web: From Conceptualization to Personalization of Contents. In Media and Metamedia Management (pp. 419-427). Springer International Publishing. 